\title{
Annular multiphase flow behavior during deep water drilling and the effect of hydrate phase transition
}

\author{
Wang Zhiyuan and Sun Baojiang* \\ School of Petroleum Engineering, China University of Petroleum (East China), Dongying, Shandong 257061, China
}

\begin{abstract}
It is very important to understand the annular multiphase flow behavior and the effect of hydrate phase transition during deep water drilling. The basic hydrodynamic models, including mass, momentum, and energy conservation equations, were established for annular flow with gas hydrate phase transition during gas kick. The behavior of annular multiphase flow with hydrate phase transition was investigated by analyzing the hydrate-forming region, the gas fraction in the fluid flowing in the annulus, pit gain, bottom hole pressure, and shut-in casing pressure. The simulation shows that it is possible to move the hydrate-forming region away from sea floor by increasing the circulation rate. The decrease in gas volume fraction in the annulus due to hydrate formation reduces pit gain, which can delay the detection of well kick and increase the risk of hydrate plugging in lines. Caution is needed when a well is monitored for gas kick at a relatively low gas production rate, because the possibility of hydrate presence is much greater than that at a relatively high production rate. The shut-in casing pressure cannot reflect the gas kick due to hydrate formation, which increases with time.
\end{abstract}

Key words: Annular multiphase flow, phase transition, natural gas hydrate, gas kick

\section{Introduction}

As oil from shallow water is increasingly depleted, the future development of deep water reserves is becoming important to oil companies. However, when drilling in oiland gas-bearing formations, the gas released from reservoirs may flow into the well bore and form gas hydrates due to the low temperature and high pressure of deep water drilling. The gas hydrates will travel with drilling fluids upwards and dissociate into gas and water when the fluids leave the hydrate stability region. This makes the annular flow of drilling and completion fluids more complicated, and brings new problems and challenges to drilling operation and well control (Barker and Gomez, 1989; Ebeltoft et al, 1997; Fossil and Sangesland, 2004). Many annular multiphase flow models has been built from the 1960s and great progress has been made. The first mathematical model of kick circulation was proposed by LeBlanc and Lewis (1968). This model was built on monophasic flow, and annular friction loss and the slippage velocity between gas and mud were not taken into account. Santos (1982) presented a mathematical model of kick circulation in deepwater, into which the slippage between gas and drilling fluid, the friction losses in the two-phase region and the void fraction were introduced. The model also considered a pattern of bubbles in the twophase region and a constant well geometry. Nickens (1987) developed a multiphase flow model based on the equations of change for mass, and momentum. This model also considered

*Corresponding author. email: sunbj1128@126.com

Received July 11, 2008 the effects of the well geometry, drillstring, bit, mud pumps, and the coupling between wellbore and reservoir. The model built by Nunes et al (2002) was analytical, with an iterative procedure to calculate the pressure distribution and gas/liquid fractions in the annulus and choke line, at each time step for a constant flow rate. However, the calculation equations were based on slug flow, which was suitable for a large gas kick. In fact the flow pattern at the annulus bottom is bubble flow and it is more suitable to consider the influx as bubble flow. Although great progress has been made in gas kick simulation, none of these models considers the influence of gas hydrate phase transition on annular multiphase flow behavior, which plays an important role in deepwater drilling. Based on the characteristics of deep water drilling, the multiphase governing equations were established when gas hydrate phase transition exists during gas kick circulation and a preliminary study was made of influence of hydrate phase transition on annular multiphase flow .

\section{Multiphase governing equations}

In deep water drilling operations, the gas released from reservoirs makes annular flow of drilling fluids more complicated, and the flow in the annulus is three-phase. Meanwhile, gas hydrates can form in the annulus due to the low temperature and high pressure in deep water. Reduced hydrostatic pressure and increased temperature can cause hydrates to dissociate into free gas and water as the gas hydrates flow upwards through the annulus. Therefore, the fluid in the annulus is a commingled multiphase, multicomponent system with phase transition as well. 


\subsection{Mass conservation equations}

The mass conservation equations of gas, drilling fluids, hydrates and cuttings are as follows:

$$
\begin{aligned}
& \frac{\partial}{\partial t}\left(A \rho_{\mathrm{g}} E_{\mathrm{g}}\right)+\frac{\partial}{\partial s}\left(A \rho_{\mathrm{g}} v_{\mathrm{g}} E_{\mathrm{g}}\right)=q_{\mathrm{g}}-A x_{\mathrm{g}} r_{\mathrm{H}} \\
& \frac{\partial}{\partial t}\left(A \rho_{\mathrm{m}} E_{\mathrm{m}}\right)+\frac{\partial}{\partial s}\left(A \rho_{\mathrm{m}} v_{\mathrm{m}} E_{\mathrm{m}}\right)=-A\left(1-x_{\mathrm{g}}\right) r_{\mathrm{H}} \\
& \frac{\partial}{\partial t}\left(A \rho_{\mathrm{H}} E_{\mathrm{H}}\right)+\frac{\partial}{\partial s}\left(A \rho_{\mathrm{H}} E_{\mathrm{H}} v_{\mathrm{H}}\right)=A r_{\mathrm{H}} \\
& \frac{\partial}{\partial t}\left(A \rho_{\mathrm{c}} E_{\mathrm{c}}\right)+\frac{\partial}{\partial s}\left(A \rho_{\mathrm{c}} E_{\mathrm{c}} v_{\mathrm{c}}\right)=q_{\mathrm{c}}
\end{aligned}
$$

where $A$ is the cross-sectional area of the annulus; $E_{\mathrm{g}}, E_{\mathrm{H}}, E_{\mathrm{c}}$, and $E_{\mathrm{m}}$ are the volume fractions of gas, hydrates, cuttings, and drilling fluids, respectively; $v_{\mathrm{g}}, v_{\mathrm{H}}, v_{\mathrm{c}}$, and $v_{\mathrm{m}}$ are the return velocities of gas, hydrates, cuttings, and drilling fluids, respectively, $\mathrm{m} / \mathrm{s} ; \rho_{\mathrm{g}}, \rho_{\mathrm{H}}, \rho_{\mathrm{c}}$, and $\rho_{\mathrm{m}}$ are the densities of gas, hydrates, cuttings, and drilling fluids, respectively, $\mathrm{kg} / \mathrm{m}^{3} ; x_{\mathrm{g}}$ is gas mass fraction in the hydrates; $q_{\mathrm{g}}$ and $q_{\mathrm{c}}$ are the production rates of gas and cuttings, respectively, $\mathrm{kg} / \mathrm{s} ; r_{\mathrm{H}}$ is the hydrate decomposition/formation rate, $\mathrm{kg} / \mathrm{s}$.

\subsection{Momentum conservation equation}

The momentum conservation equation of gas, liquid, and solid multiphase flow is deduced according to the law of conservation of momentum. The total derivative of momentum with respect to time in the unit is composed of two parts. One part is equivalent to the local derivative, which is the rate of change of the total momentum of all fluids with respec to time in the unit, and the other is equivalent to the convective derivative, which is the momentum difference between inlet and outlet within unit time. So the momentum conservation equation is:

$$
\begin{aligned}
& \frac{\mathrm{d}}{\mathrm{d} t}\left(A E_{\mathrm{g}} \rho_{\mathrm{g}} v_{\mathrm{g}}+A E_{\mathrm{m}} \rho_{\mathrm{m}} v_{\mathrm{m}}+A E_{\mathrm{H}} \rho_{\mathrm{H}} v_{\mathrm{H}}+A E_{\mathrm{c}} \rho_{\mathrm{c}} v_{\mathrm{c}}\right) \\
& +\frac{\mathrm{d}}{\mathrm{d} s}\left(A E_{\mathrm{g}} \rho_{\mathrm{g}} v_{\mathrm{g}}^{2}+A E_{\mathrm{m}} \rho_{\mathrm{m}} v_{\mathrm{m}}^{2}+A E_{\mathrm{H}} \rho_{\mathrm{H}} v_{\mathrm{H}}^{2}+A E_{\mathrm{c}} \rho_{\mathrm{c}} v_{\mathrm{c}}^{2}\right) \\
& +A g \cos \theta\left(E_{\mathrm{g}} \rho_{\mathrm{g}}+E_{\mathrm{m}} \rho_{\mathrm{m}}+E_{\mathrm{H}} \rho_{\mathrm{H}}+E_{\mathrm{c}} \rho_{\mathrm{c}}\right)+\frac{\mathrm{d}(A p)}{\mathrm{d} s}+A\left|\frac{\mathrm{d} F_{\mathrm{r}}}{\mathrm{d} s}\right| \\
& =0
\end{aligned}
$$

where $F_{\mathrm{r}}$ is friction loss, $\mathrm{Pa} ; \theta$ is well deviation angle, ${ }^{\circ} ; p$ is annular pressure, $\mathrm{Pa}$.

\subsection{Energy conservation equations}

The heat exchange process is unstable when the gas released from gas and oil reservoirs enters the wellbore. Gas hydrate formation releases heat but hydrate dissociation absorbs heat (Handa, 1986; Loevois et al, 1990; Rueff et al, 1988). Therefore, the hydrate phase transition should be taken into account when the conservation of energy equations are established. Gas and liquid two-phase energy equations are built up according to the conservation of energy.
Energy conservation equation in the annulus:

$$
\begin{aligned}
& \frac{\partial}{\partial t}\left[\rho_{\mathrm{g}} E_{\mathrm{g}}\left(h+\frac{1}{2} v_{\mathrm{g}}{ }^{2}-g \cos \theta\right)+\rho_{\mathrm{l}} E_{\mathrm{l}}\left(h+\frac{1}{2} v_{1}^{2}-g \cos \theta\right)\right] A \\
& -\left[\frac{\partial\left(w_{\mathrm{g}}\left(h+\frac{1}{2} v_{\mathrm{g}}{ }^{2}-g \cos \theta\right)\right)}{\partial s}+\frac{\partial\left(w_{1}\left(h+\frac{1}{2} v_{1}^{2}-g \cos \theta\right)\right)}{\partial s}\right] \\
& \left.+\frac{r_{\mathrm{H}} \cdot A \Delta H_{\mathrm{H}}}{M_{\mathrm{H}}}\right] \\
& =2\left[\frac{1}{A^{\prime}}\left(T_{\mathrm{ei}}-T_{\mathrm{a}}\right)-\frac{1}{B^{\prime}}\left(T_{\mathrm{a}}-T_{\mathrm{t}}\right)\right]
\end{aligned}
$$

Energy conservation equation in the drill pipe:

$$
\begin{aligned}
& \frac{\partial}{\partial t}\left(\rho_{1} E_{1}\left(h+\frac{1}{2} v_{1}^{2}-g \cos \theta\right) A_{\mathrm{t}}\right)+\frac{\partial\left(w_{1}\left(h+\frac{1}{2} v_{1}^{2}-g \cos \theta\right)\right)}{\partial s} \\
& =\frac{2}{B^{\prime}}\left(T_{\mathrm{a}}-T_{\mathrm{t}}\right)
\end{aligned}
$$

where $w_{\mathrm{g}}$ and $w_{1}$ are the mass flow rates of gas and liquid phases, respectively, $\mathrm{kg} / \mathrm{s} ; h$ is enthalpy, including intrinsic energy and kinetic energy, $\mathrm{J} ; E_{1}$ is the volume fraction of liquid; $v_{1}$ is liquid velocity, $\mathrm{m} / \mathrm{s} ; A_{\mathrm{t}}$ is the cross-sectional area of drill pipe, $\mathrm{m}^{2} ; T_{\mathrm{a}}, T_{\mathrm{ei}}$, and $T_{\mathrm{t}}$ are temperatures in the annulus, formation/sea water, and drill pipe, respectively, ${ }^{\circ} \mathrm{C}$; $M_{\mathrm{H}}$ is the average molecular weight of hydrates, $\mathrm{kg} / \mathrm{mol} ; \Delta H_{\mathrm{H}}$ is hydrate decomposition heat, $\mathrm{J} / \mathrm{mol}$.

$A^{\prime}$ and $B^{\prime}$ can be expressed as follows:

$$
\begin{aligned}
& A^{\prime}=\frac{1}{2 \pi}\left[\frac{k_{\mathrm{e}}+r_{\mathrm{co}} U_{\mathrm{a}} T_{\mathrm{D}}}{r_{\mathrm{co}} U_{\mathrm{a}} k_{\mathrm{e}}}\right] \\
& B^{\prime}=\frac{1}{2 \pi r_{\mathrm{ti}} U_{\mathrm{t}}}
\end{aligned}
$$

where $T_{\mathrm{D}}$ is dimensionless temperature (Hasan and Kabir, 1994); $U_{\mathrm{a}}$ and $U_{\mathrm{t}}$ are overall heat transfer coefficients in the annulus and drill pipe, respectively, $\mathrm{W} /\left(\mathrm{m} \cdot{ }^{\circ} \mathrm{C}\right) ; k_{\mathrm{e}}$ is heat conductivity of formation or sea water, $\mathrm{W} /\left(\mathrm{m} \cdot{ }^{\circ} \mathrm{C}\right) ; r_{\mathrm{co}}$ is the outside diameter of return line, $\mathrm{m} ; r_{\mathrm{ti}}$ is the inside diameter of drilling pipe, $\mathrm{m}$.

\section{Solution of equations}

\subsection{Definite conditions}

Boundary conditions of gas kicking are as follows:

$$
p(0, t)=p_{\mathrm{s}}, \quad q_{\mathrm{g}}(H, t)=q_{\mathrm{g}}, \quad q_{\mathrm{c}}(H, t)=q_{\mathrm{c}}
$$

where $p_{\mathrm{s}}$ is atmospheric pressure, $\mathrm{Pa} ; H$ is well depth, $\mathrm{m}$.

The inlet temperature in the drill string can be measured. The boundary condition is:

$$
T_{\mathrm{t}}(0, t)=T_{\text {in }}
$$


The temperature in the drill string is the same as that at bottom hole of the annulus, then:

$$
T_{\mathrm{t}}(H, t)=T_{\mathrm{a}}(H, t)
$$

where $T_{\mathrm{m}}$ is inlet temperature in drill string, ${ }^{\circ} \mathrm{C}$.

\subsection{Discrete solution of model}

It is difficult to achieve analytical solutions of the governing equations for multiphase flow, so a numerical computational method is recommended to solve these equations. Solving steps are as follows:

(1) Estimate the pressure of node $j$ at time $n+1$;

(2) Calculate the temperatures in the drill string and the annulus of node $j$ at time $n+1$;

(3) Confirm whether hydrates form at the above-estimated pressure and temperature by using hydrate formation thermodynamic equation, and then calculate the formation rate (Dholabhai et al, 1993; Vysniauskas and Bishnoi, 1983) or decomposition rate of hydrate (Jamaluddin et al, 1989; Kim et al, 1987);

(4) Calculate the density and other physical property parameters;

(5) Estimate the volume fractions of gas, liquid, and solid phases at node $j$ at time $n+1$; and compute phase velocities of gas, liquid, and solid with governing equations of multiphase flow;

(6) Calculate phase volume fractions, if the error is within the allowed limits, go to next step; otherwise return to (5);

(7) Substitute the parameters into the momentum equation, and calculate the new pressure at note $j$ at time $n+1$. If the error is within the allowed limits, the estimated pressure is correct in Step (1) and take the parameters at node $j$ as data of node $j+1$. Otherwise return to Step (1).

\section{Analysis of annular multiphase flow behavior}

In the case of the presence of hydrate phase, the behavior of multiphase flow in a specific well was investigated by analyzing hydrate-forming region, gas fraction in the fluid flowing in the annulus, pit gain, bottom hole pressure, and shut-in casing pressure. The basic data of the well are listed in Table 1.

Table 1 Basic data

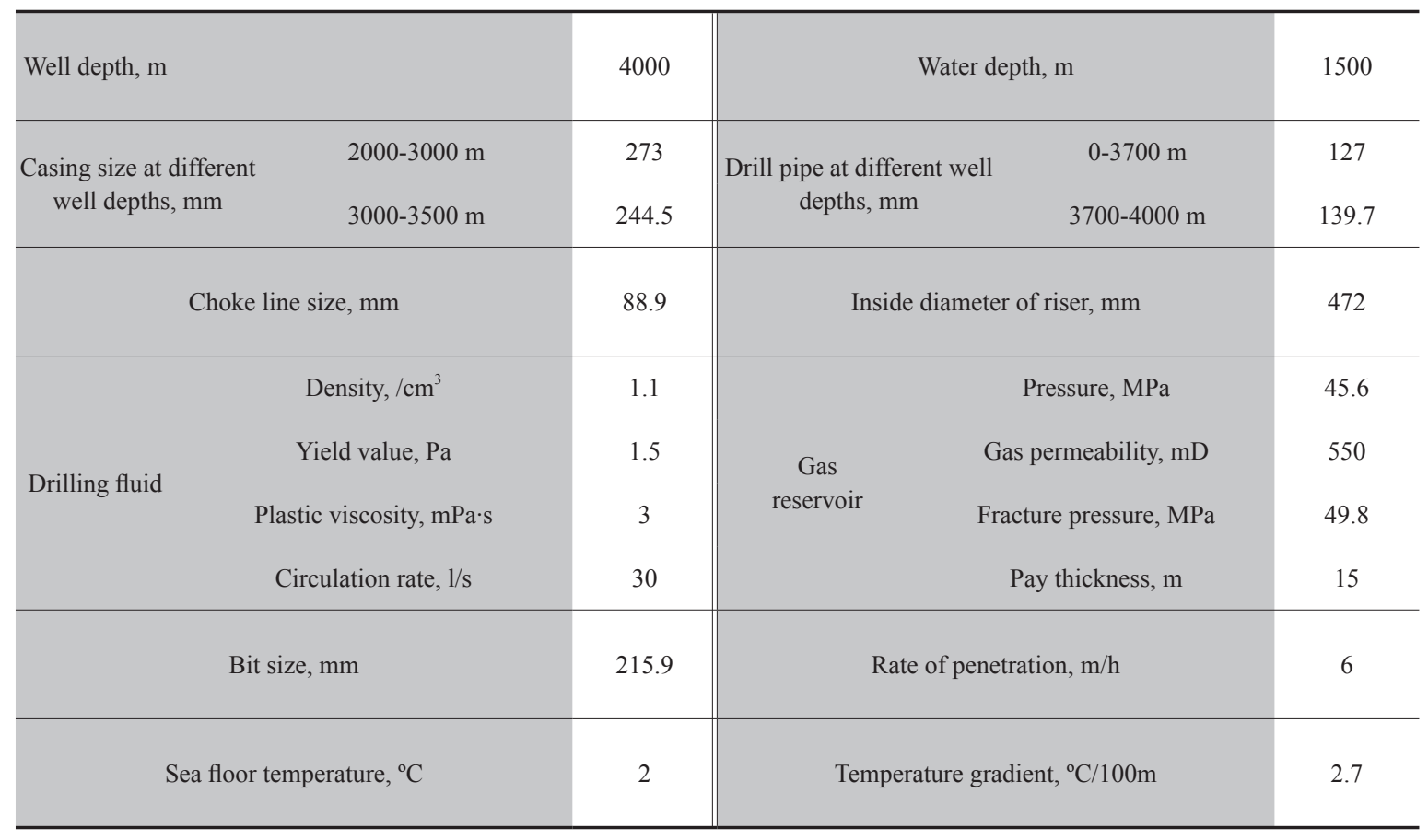

\subsection{Hydrate-forming region during drilling}

The hydrate-forming region needs to be defined in order to study the influence of hydrate phase transition on the behavior of annular multiphase flow. Hydrate-forming regions at different circulation rates during drilling are shown in Fig. 1. The region enclosed by the phase state curve and annular temperature curve is the region, in which gas hydrates are formed easily. This indicates the depth range and the temperature range (i.e. overcooled temperature) of hydrate formation.

Wellbore temperature near the mud line decreases with decreasing circulation rate. The wellbore temperature becomes close to the ambient temperature with a decrease of circulation rate due to long-term heat exchange. Moreover the hydrate-forming region becomes small when the circulation rate increases. This means that an increasing circulation rate is helpful in suppressing hydrate formation. The most favorable place for hydrate accumulation is near the blowout preventer (BOP) and it is possible to inhibit the formation of gas hydrates in this place by increasing the circulation rate, thus preventing BOP lines from being plugged with hydrate. 


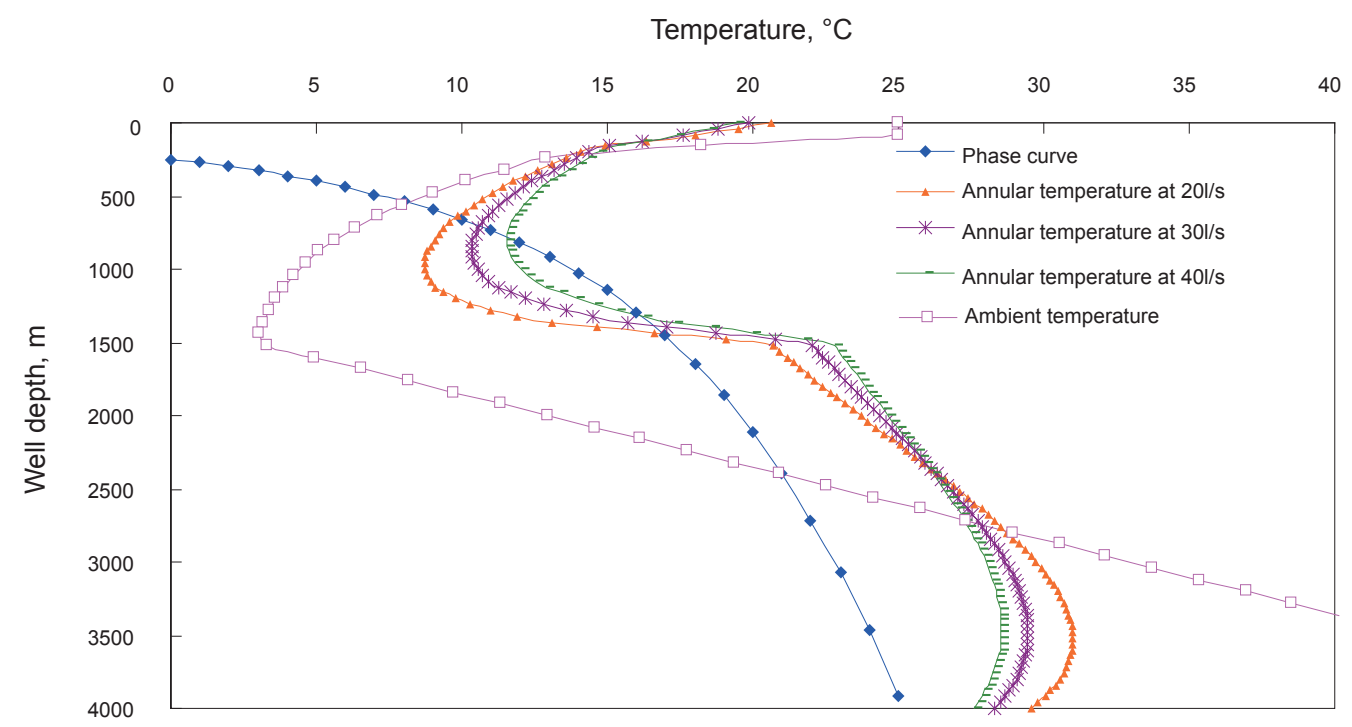

Fig. 1 Hydrate-forming regions at different circulation rates

\subsection{Gas volume fraction in the annulus during gas kick}

Fig. 2 shows gas volume fraction profiles in the annulus at different times after gas kick. Fig. 2 shows that the gas volume fraction increases with time for the fluid system, with or without hydrate phase transition, in the annulus and the hydrate phase transition has an increasingly significant effect on gas fraction with time. Meanwhile the amount of hydrates formed in the region increases gradually. The sharp decreases in gas fraction at $3,700 \mathrm{~m}$ and $1,500 \mathrm{~m}$ are due to the change of annular cross-sectional area. Below $3,700 \mathrm{~m}$ a drill collar is used to supply the proper amount of force onto the drill bit and a riser is located at above $1,500 \mathrm{~m}$, therefore at the depths of $3,700 \mathrm{~m}$ and $1,500 \mathrm{~m}$ the annular cross-sectional areas increase significantly.

Fig. 1 shows that gas hydrates are formed at a depth of $600-1,500 \mathrm{~m}$ when the circulation rate is $30 \mathrm{l} / \mathrm{s}$. The gas kick does not enter the hydrate-forming region in 20 min after the gas kick, so no gas hydrates are formed in the annulus. The gas kick enters the hydrate-forming region after $30 \mathrm{~min}$ and the gas fraction decreases gradually due to hydrate formation, compared with the volume fraction of gas without phase transition. When the annulus fluids flow close to the surface (after 60-70 min), the gas in fluids expands significantly due to pressure drop so the gas volume fraction increases sharply, and the volume fractions of gas with and without phase transition are similar.

The decrease in gas volume fraction reduces pit gain, which can delay the detection of well kick. What is worse is that the hydrates formed in the annulus can plug lines.

Fig. 3 shows gas volume fraction profiles at different gas production rates at 30 min during gas kick circulation. The gas frontals flow into the hydrate-forming region earlier when producing at a higher gas rate and the gas volume fraction in the annulus is much larger than those producing at a lower gas rate. Therefore, pit gain rises with increasing gas production rate, which makes it much easier to detect gas kick in reservoirs with a large gas production rate. This case should be paid more attention to when gas kick is monitored at a low gas production rate because the possibility of hydrate formation is much greater when producing at a low gas rate.

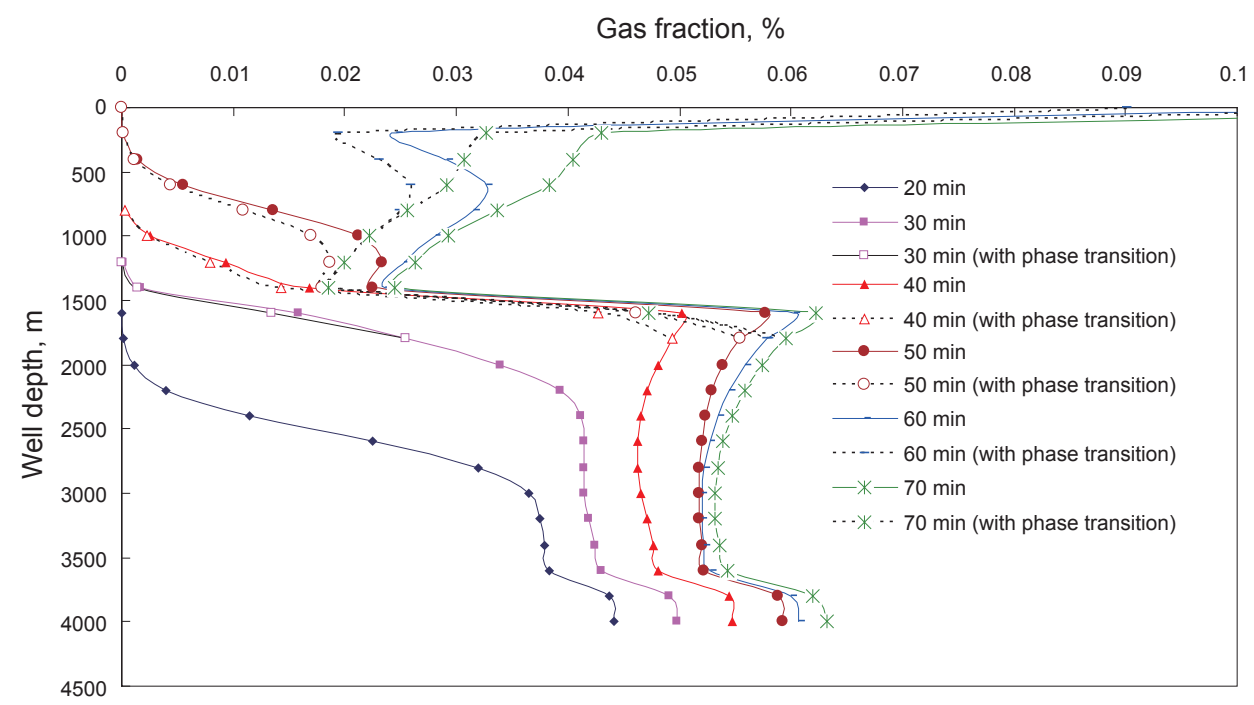

Fig. 2 Comparison of gas fraction profiles with/without hydrate phase transition during gas kick (at circulation rate of 30 l/s) 


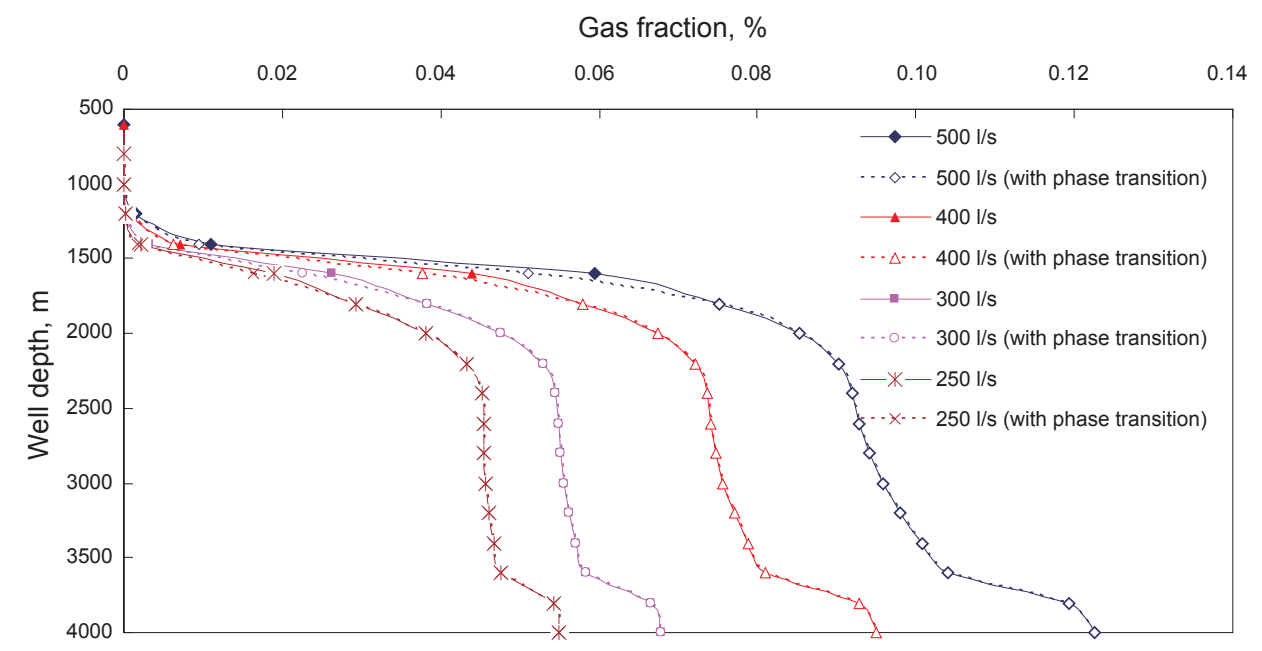

Fig. 3 Gas fraction profiles at different gas production rates at 30 min during gas kick

\subsection{Pit gain during gas kick}

Fig. 4 shows pit gain variation with time under different formation permeabilities. Simulation indicates that the pit gain is an exponential function of time. A possible explanation is that the inflow of formation fluid (gas) into the well bore makes bottom hole pressure decline gradually. This leads to an increase in the difference between bottom hole pressure and formation pressure, so gas kick volume increases gradually (more gas will flow into the well bore). However, after 50 minutes, the pit gain increases slowly, because the gas has arrived at the surface and the escaped gas cannot contribute to the growth of pit gain. After 20 more minutes the gas kick enters the hydrate-forming region and hydrate formation makes the gas fraction decrease. A comparison of pit gain shows that the presence of hydrate phase may reduce pit gain. This brings great challenges to early kick detection during deepwater drilling. Several improvements are required to optimize available detection methods in order to recognize the gas kick in time.

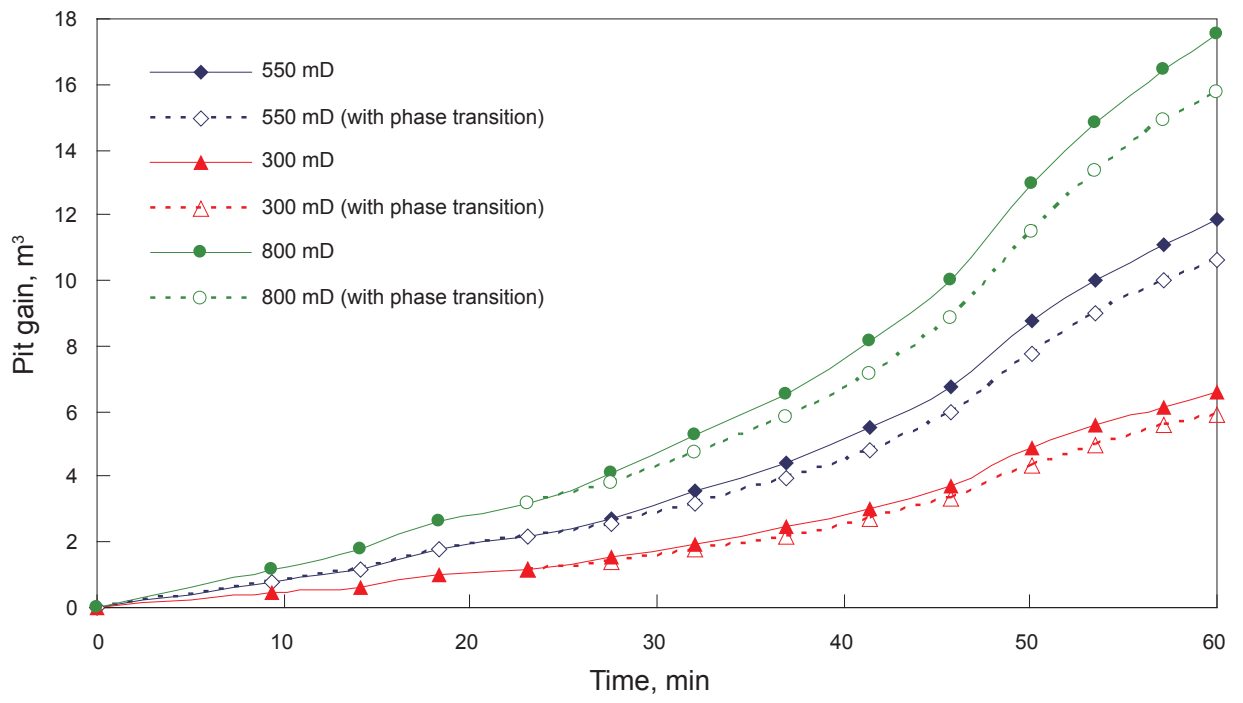

Fig. 4 Pit gain during gas kick under different formation permeabilities

\subsection{Bottom hole pressure during gas kick}

Fig. 5 shows bottom hole pressure versus time during gas kick. When the gas released from gas and oil reservoirs flows into the wellbore, the bottom hole pressure changes slowly at the beginning stage, but declines rapidly with time. The bottom hole pressure is a result of hydrostatic pressure exerted by drilling fluids and friction loss of return fluids in the annulus. More and more gas enters the wellbore with time, which reduces the hydrostatic pressure. However, the influx of gas accelerates the annular fluid velocity and then the friction loss increases correspondingly. The bottom hole pressure drops gradually with time because the increment in friction loss is much less than the decrement in hydrostatic pressure, Meanwhile the decline in bottom hole pressure enhances pressure difference, which accelerates gas production. When the gas flows through the riser after 20 minutes, the decrease in hydrostatic pressure tends to slow down due to the large cross-sectional area of riser. After 50 minutes, some gas has escaped from wellbore and cannot cause hydrostatic pressure decline, the bottom hole pressure tends to decline relatively slowly. Therefore the bottom hole pressure curve shows nonlinear variation with different stages. 


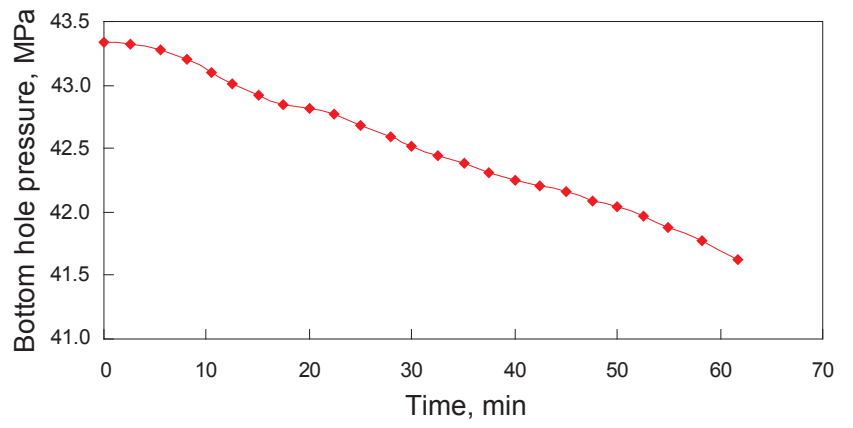

Fig - 5 Bottom pressure during gas kick (without hydrate phase transition)

In addition, hydrate-forming region is mainly above the mud line. Because the inside diameter of the riser is much larger than that of the annulus and the volume of gas consumed during hydrate formation is low, gas hydrate formation can be neglected compared with the relatively large volume of riser. Therefore, the bottom pressure is not seriously affected by hydrate formation.

\subsection{Shut-in casing pressure during gas kick}

Fig. 6 shows a plot of shut-in casing pressure with time for different permeability reservoirs. The gas released from gas and oil reservoirs reduces the bottom hole pressure, which cannot balance formation pressure, so the casing pressure will build up after well shut in. More and more gas enters the well bore with time and the hydrostatic loss becomes serious, therefore the shut-in casing pressure increases with time. The variation of shut-in casing pressure is due to the same reason for bottom hole pressure variation, the shut-in casing pressure curve also shows nonlinear variation with different stages. The gas fraction declines due to hydrate formation, which makes the hydrostatic pressure increase slightly and the shutin casing pressure drop slightly.

Thus, the behavior of multiphase flow with hydrate phase transition was investigated by analyzing the hydrateforming region, the gas fraction in the fluid flowing in the annulus, pit gain, bottom hole pressure and shut-in casing pressure.

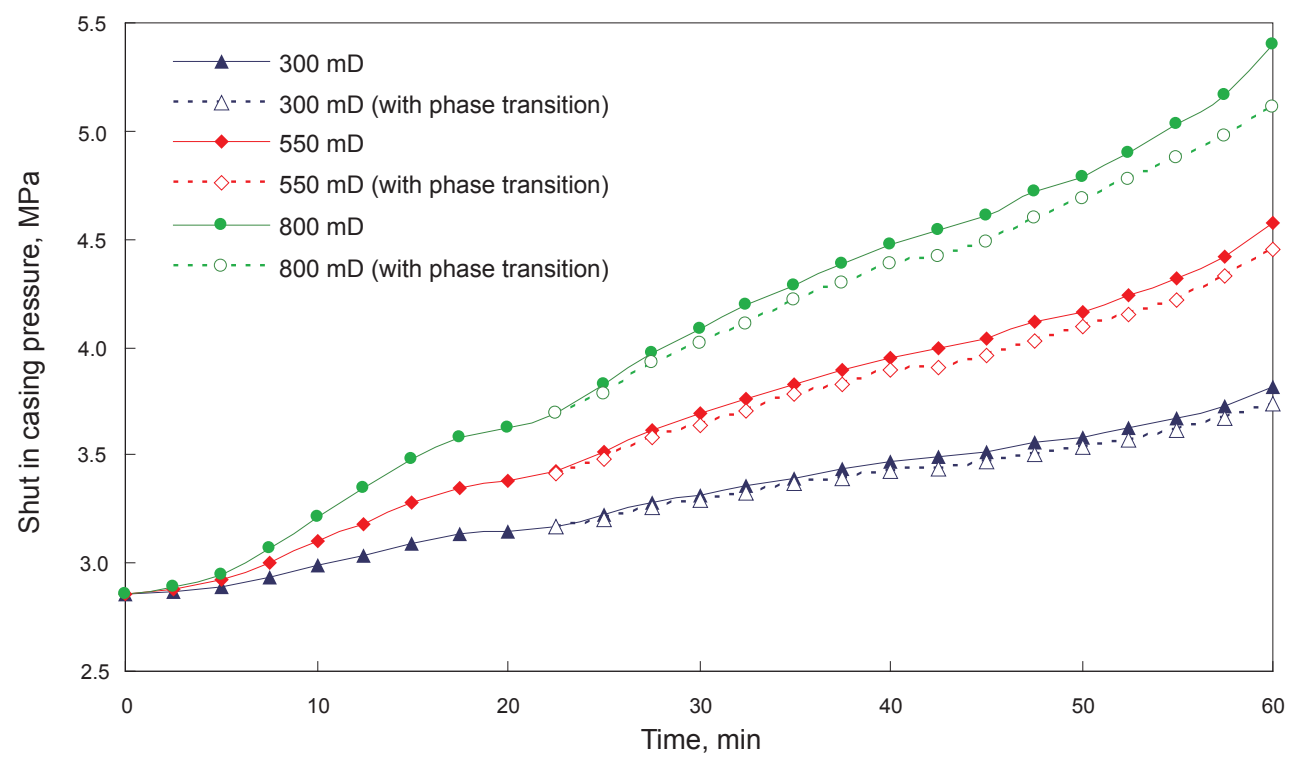

Fig. 6 Shut-in casing pressure during gas kick

\section{Conclusions}

1) The hydrate-forming region becomes small with increasing circulation rate and it is possible to make the hydrate-forming region away from sea floor by increasing circulation rate, thus preventing BOP lines from hydrate plugging.

2) The decrease in gas volume fraction in the annulus due to hydrate formation reduces pit gain, which can delay the detection of well kick and increase the risk of hydrate plugging in lines, so early detection of well kick should be strengthened during deepwater drilling.

3) Caution is needed in gas kick monitoring when the gas production rate is relatively low, because the possibility of hydrate formation is much greater than that at a relatively higher production rate.

4) Shut-in casing pressure cannot truly reflect a gas kick due to hydrate formation which increases with time. So hydrate phase transition should be taken into account when the extent of gas kick is determined according to shut-in casing pressure.

\section{Acknowledgements}

The authors are grateful to the efforts of Cheng Haiqing and Dr. Gao Yonghai. This work is supported by the China National 863 Program (Grant No. 2006AA09A106), the Doctoral Program of Higher Education of China (Grant No. 20060425502), the National Natural Science Foundation of China (Grant No.50874116) and Shandong Province Natural Science Foundation(Grant No.Z2007A01). 


\section{References}

Barker J W and Gomez R K. Formation of hydrates during deepwater drilling operations. Journal of Petroleum Technology. 1989. 41(3): 297-301

Dholabhai P D, Kalogerakis N and Bishnoi P R. Kinetics of methane hydrate formation in aqueous electrolyte solutions. Canadian Journal of Chemical Engineering. 1993. 71(2): 68-74

Ebeltoft H, Yousif M and Soergaard E. Hydrate control during deep water drilling: overview and new drilling fluids. SPE Annual Technical Conference and Exhibition, 5-8 October 1997, San Antonio, Texas (SPE 38567)

Fossil B and Sangesland S. Managed pressure drilling for subsea applications: well control challenges in deep waters. SPE/IADC Underbalanced Technology Conference and Exhibition, 11-12 October 2004, Houston, Texas (SPE/IADC 91633)

Handa Y P. Compositions enthalpies of dissociation, and heat capacities in the range 85 to $270 \mathrm{~K}$ for clathrate hydrates of $\mathrm{CH}_{4}, \mathrm{C}_{2} \mathrm{H}_{6}, \mathrm{C}_{3} \mathrm{H}_{8}$. Journal of Chemical Thermodynamics. 1986. 18(7): 915-921

Hasan A R and Kabir C S. Aspects of wellbore heat transfer during twophase flow. SPE Production \& Facilities. 1994. 9(4): 211-216 (SPE 22948)

Jamaluddin A M, Kalogerakis N and Bishnoi P R. Modeling of decomposition of a synthetic core of methane gas hydrate by coupling intrinsic kinetics with heat transfer rates. Phys. Chem. 1989. 67(6): $948-954$

Kim H C, Bishnoi P R and Heidemann R A. Kinetics of methane hydrate decomposition. Chemical Engineering Science. 1987. 42(7): 1645-1653.

LeBlanc J L and Lewis R L. A mathematical model of a gas kick. Journal of Petroleum Technology. 1968. 20(8): 888-898

Loevois J S, Perkins R, Martin R J, et al. Development of an automated high pressure heat flux calorimeter and its application to measure the heat of dissociation and hydrate member of methane hydrate. Fluid Phase Equilibrium. 1990. 59: 73-79

Nickens H V. A dynamic computer model of a kicking well. SPE Drilling Engineering. 1987. 7: 158-173

Nunes J O L, Bannwart A C and Ribeiro P R. Mathematical model of a gas kick in deep water scenario. IADC/SPE Asia Pacific Drilling Technology, 8-11 September 2002, Jakarta, Indonesia (SPE/IADC 77253)

Rueff R M, Sloan E D and Yesavage V F. Heat capacity and heat of dissociation of methane hydrates. AIChE J. 1988. 34(9): 1468-1476

Santos O A. A mathematical model of a gas kick when drilling in deep waters. MS Thesis. Colorado School of Mines. 1982

Vysniauskas A and Bishnoi P R. A kinetic study of methane hydrate formation. Chemical Engineering Science. 1983. 38(7): 1061-1072

(Edited by Sun Yanhua) 